\title{
Truly Nonlinear Oscillations
}

Harmonic Balance, Parameter Expansions,

Iteration, and Averaging Methods

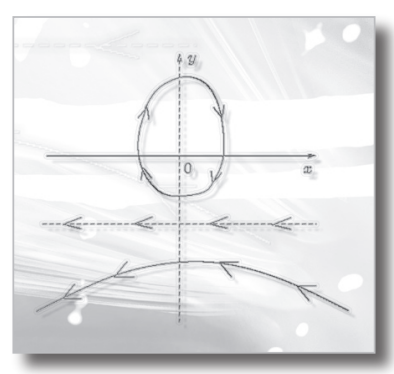


This page intentionally left blank 


\section{Truly /onlinear Oscillations}

Harmonic Balance, Parameter Expansions, Iteration, and Averaging Methods

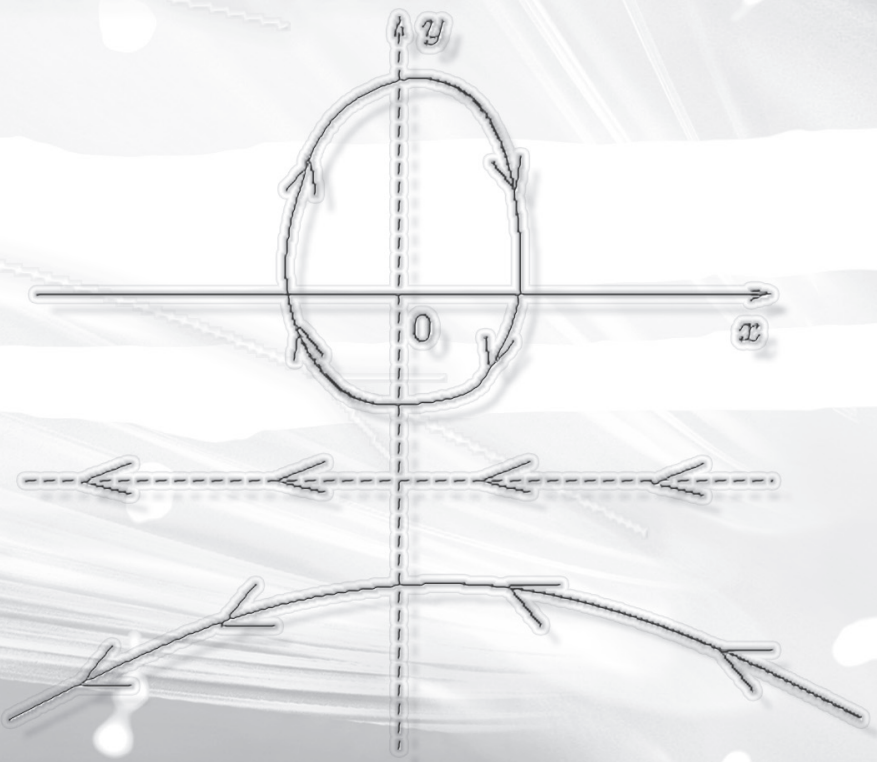

Ronald E Mickens

Clark Atlanta University, USA 


\title{
Published by
}

World Scientific Publishing Co. Pte. Ltd.

5 Toh Tuck Link, Singapore 596224

USA office: 27 Warren Street, Suite 401-402, Hackensack, NJ 07601

UK office: 57 Shelton Street, Covent Garden, London WC2H 9HE

\author{
Library of Congress Cataloging-in-Publication Data \\ Mickens, Ronald E., 1943- \\ Truly nonlinear oscillations : harmonic balance, parameter expansions, iteration, \\ and averaging methods / by Ronald E. Mickens. \\ p. $\mathrm{cm}$. \\ Includes bibliographical references and index. \\ ISBN-13: 978-981-4291-65-1 (hardcover : alk. paper) \\ ISBN-10: 981-4291-65-X (hardcover : alk. paper) \\ 1. Approximation theory. 2. Nonlinear oscillations. I. Title.
}

QA221.M53 2010

$511^{\prime} \cdot 4--\mathrm{dc} 22$

2009038794

\section{British Library Cataloguing-in-Publication Data}

A catalogue record for this book is available from the British Library.

Copyright (C) 2010 by World Scientific Publishing Co. Pte. Ltd.

All rights reserved. This book, or parts thereof, may not be reproduced in any form or by any means, electronic or mechanical, including photocopying, recording or any information storage and retrieval system now known or to be invented, without written permission from the Publisher.

For photocopying of material in this volume, please pay a copying fee through the Copyright Clearance Center, Inc., 222 Rosewood Drive, Danvers, MA 01923, USA. In this case permission to photocopy is not required from the publisher.

Printed in Singapore. 


\title{
Truly Nonlinear Oscillators
}

An Introduction to Harmonic Balance, Parameter Expansion, Iteration, and Averaging Methods

\author{
Ronald E. Mickens \\ Distinguished Fuller E. Callaway Professor \\ Clark Atlanta University \\ Atlanta, GA 30314, USA
}


This book is dedicated to my family:

Daughter ... Leah, Son ... James, Wife ... Maria. 


\section{Preface}

This small volume introduces several important methods for calculating approximations to the periodic solutions of "truly nonlinear" (TNL) oscillator differential equations. This class of equations take the form

$$
\ddot{x}+g(x)=\epsilon F(x, \dot{x}),
$$

where $g(x)$ has no linear approximation at $x=0$. During the past several decades a broad range of calculational procedures for solving such differential equations have been created by an internationally based group of researchers. These techniques appear under headings such as

- averaging

- combined and linearization

- harmonic balance

- homotopy perturbation

- iteration

- parameter expansion

- variational iteration methods.

Further, these methodologies have not only been applied to TNL oscillators, but also to strongly nonlinear oscillations where a parameter may take on large values. Most of these techniques have undergone Darwinian type evolution and, as a consequence, a large number of papers are published each year on specializations of a particular method. While we have been thorough in our personal examination of the research literature, only those papers having an immediate connection to the topic under discussion are cited because of the magnitude of existing publications and because an interested user of this volume can easily locate the relevant materials from various websites. 
We have written this book for the individual who wishes to learn, understand, and apply available techniques for analyzing and solving problems involving TNL oscillations. It is assumed that the reader of this volume has a background preparation that includes knowledge of perturbation methods for the standard oscillatory systems modeled by the equation

$$
\ddot{x}+x=\epsilon F(x, \dot{x}) \text {. }
$$

In particular, this includes an understanding of concepts such as secular terms, limit-cycles, uniformly-valid approximations, and the elements of Fourier series.

The basic style and presentation of the material in this book is heuristic rather than rigorous. The references at the end of each chapter, along with an examination of relevant websites, will allow the reader to fully comprehend what is currently known about a particular technique. However, the reader should also realize that the creation and development of most of the methods discussed in this book do not derive from rigorous mathematical derivations. This task is a future project for those who have the interests and necessary background to carry out these procedures. However, these efforts are clearly not relevant for our present needs.

The book consists of seven chapters and several appendices. Chapter 1 offers an overview of the book. In particular, it presents a definition of TNL equations, introduces the concept of odd-parity systems, and calculates the exact solutions to four TNL oscillatory systems.

Chapter 2 provides a brief discussion of several procedures for a priori determining whether a given TNL differential equation has periodic and/or oscillatory solutions. The next four chapters present introductions to most of the significant procedures for calculating analytical approximations to the solutions of TNL differential equations. These chapters discuss, respectively, harmonic balance, parameter expansion, iteration, and averaging methods. Each chapter gives not only the basic methodology for each technique, but also provides a range of worked examples illustrating their use.

The last chapter considers six TNL oscillator equations and compares results obtained by all the methods that are applicable to each. It ends with general comments on TNL oscillators and provides a short listing of unresolved research problems.

We also include a number of appendices covering topics relevant to understanding the general issues covered in this book. The topics discussed range from certain mathematical relations to basic results on linear secondorder differential equations having constant coefficients. Brief presentations 
are given on Fourier series, the Lindstedt-Poincaré perturbation method, and the standard first-order method of averaging. A final appendix, "Discrete Models of Two TNL Oscillators," illustrates the complexities that may arise when one attempts to construct discretizations to calculate numerical solutions.

I thank my many colleagues around the world for the interest in my work, their generalization of these results and their own original "creations" on the subject of TNL oscillations. As always, I am truly grateful to Ms. Annette Rohrs for her technical services in seeing that my handwritten pages were transformed into the present format. Both she and my wife, Maria Mickens, provided valuable editorial assistance and the needed encouragement to successfully complete this project. Finally, I wish to acknowledge Dr. Shirley Williams-Kirksey, Dean of the School of Arts and Sciences, for providing Professional Development Funds to assist in the completion of this project. Without this support the writing effort would not have been done on time.

Ronald E. Mickens

Atlanta, GA

August 2009 
This page intentionally left blank 


\section{Contents}

Preface vii

List of Figures $\quad$ xix

List of Tables $\quad$ xxi

1. Background and General Comments 1

1.1 Truly Nonlinear Functions . . . . . . . . . . . . . . . . . . 1

1.2 Truly Nonlinear Oscillators . . . . . . . . . . . . . 2

1.3 General Remarks . . . . . . . . . . . . . . . . . 3

1.4 Scaling and Dimensionless Form of Differential Equations 5

1.4.1 Linear Damped Oscillator . . . . . . . . . 5

1.4.2 Nonlinear Oscillator . . . . . . . . . . . . . 6

$1.4 .3 \quad \ddot{x}+a x^{p}=0 \ldots \ldots \ldots \ldots \ldots \ldots$

1.4.4 $\ddot{x}+a x+b x^{1 / 3}=0 \ldots \ldots \ldots \ldots$

1.5 Exactly Solvable TNL Oscillators . . . . . . . . . . 9

1.5.1 Antisymmetric, Constant Force Oscillator . . . . . 10

1.5.2 Particle-in-a-Box . . . . . . . . . . . . . 11

1.5.3 Restricted Duffing Equation . . . . . . . . . . 12

1.5.4 Quadratic Oscillator . . . . . . . . . . . . . 14

1.6 Overview of TNL Oscillator Methods . . . . . . . . . . . . 14

1.6.1 Harmonic Balance . . . . . . . . . . . . . . . . 16

1.6.2 Parameter Expansion . . . . . . . . . . . . 16

1.6.3 Averaging Methods . . . . . . . . . . . . . . . 17

1.6.4 Iteration Techniques . . . . . . . . . . . . . . . . . 18

1.7 Discussion . . . . . . . . . . . . . . . . . . . . 18

Problems . . . . . . . . . . . . . . . . 20 
References ...................... 21

2. Establishing Periodicity 23

2.1 Phase-Space . . . . . . . . . . . . . . . . . . 23

2.1.1 System Equations . . . . . . . . . . . . . . . . . . . . . . . 24

2.1.2 Fixed-Points . . . . . . . . . . . . . . . 24

2.1.3 ODE for Phase-Space Trajectories . . . . . . . . 25

2.1.4 Null-clines . . . . . . . . . . . . . . . . 25

2.1.5 Symmetry Transformations . . . . . . . . . . 26

2.1.6 Closed Phase-Space Trajectories . . . . . . . . . . 26

2.1.7 First-Integrals . . . . . . . . . . . . . . 26

2.2 Application of Phase-Space Methods . . . . . . . . . . . . . 27

2.2.1 Linear Harmonic Oscillator . . . . . . . . . . . 27

2.2.2 Several TNL Oscillator Equations . . . . . . . . 31

2.3 Dissipative Systems: Energy Methods . . . . . . . . . . 33

2.3.1 Damped Linear Oscillator . . . . . . . . . . 35

2.3.2 Damped TNL Oscillator . . . . . . . . . . 35

2.3.3 Mixed-Damped TNL Oscillator . . . . . . . . 36

2.4 Resume ....................... 39

Problems . . . . . . . . . . . . . . . . . 39

References ................... 40

3. Harmonic Balance 43

3.1 Direct Harmonic Balance: Methodology . . . . . . . . . . 44

3.2 Worked Examples . . . . . . . . . . . . . 46

$3.2 .1 \quad \ddot{x}+x^{3}=0 \ldots \ldots \ldots \ldots \ldots \ldots$

$3.2 .2 \quad \ddot{x}+x^{-1}=0 \ldots \ldots \ldots \ldots \ldots$

$3.2 .3 \quad \ddot{x}+x^{2} \operatorname{sgn}(x)=0 \ldots \ldots \ldots \ldots \ldots$

$3.2 .4 \quad \ddot{x}+x^{1 / 3}=0 \ldots \ldots \ldots \ldots \ldots \ldots$

$3.2 .5 \quad \ddot{x}+x^{-1 / 3}=0 \ldots \ldots \ldots \ldots \ldots \ldots$

3.3 Rational Approximations . . . . . . . . . . . . 61

3.3.1 Fourier Expansion . . . . . . . . . . . . . 62

$3.3 .2 \quad$ Properties of $a_{k} \ldots \ldots \ldots \ldots \ldots$

3.3.3 Calculation of $\ddot{x} \ldots \ldots \ldots$. . . . . . . . 63

3.4 Worked Examples . . . . . . . . . . . . . 63

$3.4 .1 \quad \ddot{x}+x^{3}=0 \ldots \ldots \ldots \ldots \ldots \ldots \ldots$

$3.4 .2 \quad \ddot{x}+x^{2} \operatorname{sgn}(x)=0 \ldots \ldots \ldots \ldots \ldots$

3.4.3 $\ddot{x}+x^{-1}=0 \ldots \ldots \ldots \ldots \ldots 6$ 
3.5 Third-Order Equations . . . . . . . . . . . . . . 67

3.5.1 Castor Model . . . . . . . . . . . . . . . 68

3.5.2 TNL Castor Models . . . . . . . . . . . . . . 69

3.6 Resume ....................... 70

3.6 .1 Advantages . . . . . . . . . . . . . 70

3.6 .2 Disadvantages . . . . . . . . . . . . . 70

Problems ......................... 71

References ....................... 72

4. Parameter Expansions $\quad 75$

4.1 Introduction . . . . . . . . . . . . . . 75

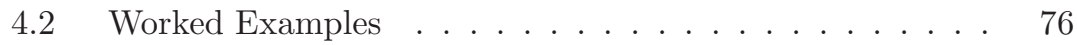

$4.2 .1 \quad \ddot{x}+x^{3}=0 \ldots \ldots \ldots \ldots \ldots$

$4.2 .2 \quad \ddot{x}+x^{-1}=0 \ldots \ldots \ldots \ldots \ldots \ldots$

$4.2 .3 \quad \ddot{x}+x^{3} /\left(1+x^{2}\right)=0 \ldots \ldots \ldots$. . . . . 80

$4.2 .4 \quad \ddot{x}+x^{1 / 3}=0 \ldots \ldots \ldots \ldots \ldots$. . . . . . . 81

$4.2 .5 \quad \ddot{x}+x^{3}=\epsilon\left(1-x^{2}\right) \dot{x} \ldots \ldots \ldots \ldots \ldots$

$4.2 .6 \quad \ddot{x}+\operatorname{sgn}(x)=0 \ldots \ldots \ldots \ldots \ldots$

4.3 Discussion . . . . . . . . . . . . . . . 86

4.3.1 Advantages . . . . . . . . . . . . . . 87

4.3.2 Difficulties . . . . . . . . . . . . . . 87

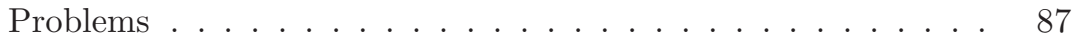

References ...................... 88

5. Iteration Methods $\quad 89$

5.1 General Methodology . . . . . . . . . . . . . 89

5.1 .1 Direct Iteration . . . . . . . . . . . . . . . . . 89

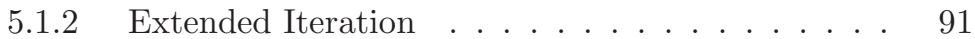

5.2 Worked Examples: Direct Iteration . . . . . . . . . . . . 92

$5.2 .1 \quad \dot{x}+x^{3}=0 \ldots \ldots \ldots \ldots \ldots 2$

$5.2 .2 \quad \ddot{x}+x^{3} /\left(1+x^{2}\right)=0 \ldots \ldots \ldots \ldots 7$

$5.2 .3 \quad \ddot{x}+x^{-1}=0 \ldots \ldots \ldots \ldots \ldots$

$5.2 .4 \quad \ddot{x}+\operatorname{sgn}(x)=0 \ldots \ldots \ldots \ldots \ldots$

$5.2 .5 \quad \ddot{x}+x^{1 / 3}=0 \ldots \ldots \ldots \ldots \ldots \ldots \ldots$

$5.2 .6 \quad \ddot{x}+x^{-1 / 3}=0 \ldots \ldots \ldots \ldots \ldots$

$5.2 .7 \quad \ddot{x}+x+x^{1 / 3}=0 \ldots \ldots \ldots \ldots \ldots$

5.3 Worked Examples: Extended Iteration . . . . . . . . . . . 112

$5.3 .1 \quad \ddot{x}+x^{3}=0 \ldots \ldots \ldots \ldots \ldots$ 
$5.3 .2 \quad \ddot{x}+x^{-1}=0 \ldots \ldots \ldots \ldots \ldots \ldots$

5.4 Discussion . . . . . . . . . . . . . . . . 117

5.4.1 Advantages of Iteration Methods . . . . . . . . . 118

5.4.2 Disadvantages of Iteration Methods . . . . . . . . 119

Problems . . . . . . . . . . . . . . . . . 120

References ..................... 121

6. Averaging Methods 123

6.1 Elementary TNL Averaging Methods . . . . . . . . . . . . 124

6.1.1 Mickens-Oyedeji Procedure . . . . . . . . . . . . 124

6.1.2 Combined Linearization and Averaging Method . 126

6.2 Worked Examples . . . . . . . . . . . . . . . . 129

$6.2 .1 \quad \ddot{x}+x^{3}=-2 \epsilon \dot{x} \ldots \ldots \ldots \ldots \ldots \ldots$

$6.2 .2 \quad \ddot{x}+x^{3}=-\epsilon \dot{x}^{3} \ldots \ldots \ldots \ldots \ldots \ldots$

$6.2 .3 \quad \ddot{x}+x^{3}=\epsilon\left(1-x^{2}\right) \dot{x} \ldots \ldots \ldots \ldots \ldots \ldots$

$6.2 .4 \quad \ddot{x}+x^{1 / 3}=-2 \epsilon \dot{x} \ldots \ldots \ldots \ldots \ldots . \ldots \ldots$

$6.2 .5 \quad \ddot{x}+x^{1 / 3}=\epsilon\left(1-x^{2}\right) \dot{x} \ldots \ldots \ldots \ldots \ldots . \ldots \ldots$

$6.2 .6 \quad \ddot{x}+x=-2 \epsilon(\dot{x})^{1 / 3} \ldots \ldots \ldots \ldots \ldots \ldots$

6.2.7 General Comments . . . . . . . . . . . . 137

6.3 Cveticanin's Averaging Method . . . . . . . . . . . . 138

6.3.1 Exact Period . . . . . . . . . . . . . . . . 139

6.3.2 Averaging Method . . . . . . . . . . . . . . . 140

6.3.3 Summary . . . . . . . . . . . . . . . . . . . . 142

6.4 Worked Examples . . . . . . . . . . . . . . . . 142

6.4.1 $\ddot{x}+x|x|^{\alpha-1}=-2 \epsilon \dot{x} \ldots \ldots \ldots \ldots \ldots . \ldots \ldots 2$

$6.4 .2 \quad \ddot{x}+x|x|^{\alpha-1}=-2 \epsilon(\dot{x})^{3} \ldots \ldots \ldots \ldots 144$

6.4.3 $\ddot{x}+x|x|^{\alpha-1}=\epsilon\left(1-x^{2}\right) \dot{x} \quad \ldots \ldots \ldots \ldots . \ldots . \ldots 145$

6.5 Chronology of Averaging Methods . . . . . . . . . . . 147

6.6 Comments . . . . . . . . . . . . . . . . . . . . . . 149

Problems . . . . . . . . . . . . . . . . . . 151

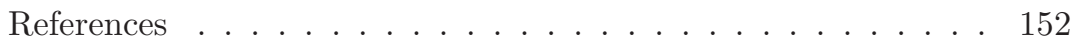

7. Comparative Analysis 155

7.1 Purpose ........................ 155

$7.2 \quad \ddot{x}+x^{3}=0 \ldots \ldots \ldots \ldots \ldots \ldots \ldots \ldots \ldots$

7.2.1 Harmonic Balance . . . . . . . . . . . . . 156

7.2 .2 Parameter Expansion . . . . . . . . . . . . . . . . 158

7.2 .3 Iteration . . . . . . . . . . . . . . . 158 
7.2.4 Comments . . . . . . . . . . . . . . 159

$7.3 \quad \ddot{x}+x^{1 / 3}=0 \ldots \ldots \ldots \ldots \ldots \ldots$

7.3.1 Harmonic Balance . . . . . . . . . . . . . . . . 160

7.3.2 Parameter Expansion . . . . . . . . . . . . . . . . 161

7.3 .3 Iteration . . . . . . . . . . . . . . . . 162

7.3.4 Comment. . . . . . . . . . . . . . 162

$7.4 \quad \ddot{x}+x^{3}=-2 \epsilon \dot{x} \ldots \ldots \ldots \ldots \ldots \ldots$

7.4 .1 Mickens-Oyedeji . . . . . . . . . . . . . 163

7.4.2 Combined-Linearization-Averaging . . . . . . 165

7.4.3 Cveticanin's Method . . . . . . . . . . . . 166

7.4 .4 Discussion . . . . . . . . . . . . . 167

$7.5 \quad \ddot{x}+x^{1 / 3}=-2 \epsilon \dot{x} \ldots \ldots \ldots \ldots \ldots 16 \ldots \ldots$

7.5.1 Combined-Linearization-Averaging . . . . . . 167

7.5.2 Cveticanin's Method . . . . . . . . . . . . 168

7.5 .3 Discussion . . . . . . . . . . . . . . 170

$7.6 \quad \ddot{x}+x^{3}=\epsilon\left(1-x^{2}\right) \dot{x} \quad \ldots \ldots \ldots \ldots \ldots \ldots$

7.6.1 Mickens-Oyedeji . . . . . . . . . . . . . . . 170

7.6 .2 Cveticanin's Method . . . . . . . . . . . . . 171

7.6 .3 Discussion . . . . . . . . . . . . . . 172

$7.7 \quad \ddot{x}+x^{1 / 3}=\epsilon\left(1-x^{2}\right) \dot{x} \ldots \ldots \ldots \ldots \ldots \ldots$

7.8 General Comments and Calculation Strategies . . . . . . . 175

7.8.1 General Comments . . . . . . . . . . . . 176

7.8 .2 Calculation Strategies . . . . . . . . . . . 177

7.9 Research Problems . . . . . . . . . . . . . . . . . . 179

References ...................... . 181

Appendix A Mathematical Relations 183

A.1 Trigonometric Relations . . . . . . . . . . . . . . . 183

A.1.1 Exponential Definitions of Trigonometric Functions 183

A.1.2 Functions of Sums of Angles . . . . . . . . . 183

A.1.3 Powers of Trigonometric Functions . . . . . . . . 183

A.1.4 Other Trigonometric Relations . . . . . . . . . 184

A.1.5 Derivatives and Integrals of Trigonometric

Functions . . . . . . . . . . . . . 185

A.2 Factors and Expansions . . . . . . . . . . . 186

A.3 Quadratic Equations . . . . . . . . . . . . . . . 187

A.4 Cubic Equations . . . . . . . . . . . . . . 187

A.5 Differentiation of a Definite Integral with Respect to a Parameter 
A.6 Eigenvalues of a $2 \times 2$ Matrix . . . . . . . . . . 188

References . . . . . . . . . . . . . . 189

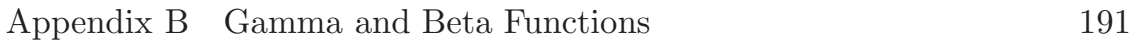

B.1 Gamma Function . . . . . . . . . . . . . . . . . . . . 191

B.2 The Beta Function . . . . . . . . . . . . . . . . . 191

B.3 Two Useful Integrals . . . . . . . . . . . . . . . . . . 192

$\begin{array}{lll}\text { Appendix C } & \text { Fourier Series } & 193\end{array}$

C.1 Definition of Fourier Series . . . . . . . . . . . . 193

C.2 Convergence of Fourier Series . . . . . . . . . . . . 194

C.2.1 Examples . . . . . . . . . . . . . . . . 194

C.2.2 Convergence Theorem . . . . . . . . . . . . . . 194

C.3 Bounds on Fourier Coefficients . . . . . . . . . . . . 195

C.4 Expansion of $F(a \cos x,-a \sin x)$ in a Fourier Series . . . 195

C.5 Fourier Series for $(\cos \theta)^{\alpha}$ and $(\sin \theta)^{\alpha} \ldots \ldots \ldots 196$

References ........................ 198

Appendix D Basic Theorems of the Theory of Second-Order Differential Equations 199

D.1 Introduction . . . . . . . . . . . . . . . . . 199

D.2 Existence and Uniqueness of the Solution . . . . . . . 200

D.3 Dependence of the Solution on Initial Conditions . . . . 200

D.4 Dependence of the Solution on a Parameter . . . . . . . . 201

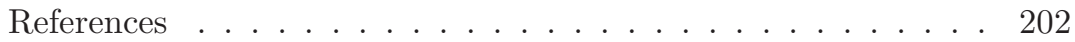

Appendix E Linear Second-Order Differential Equations 203

E.1 Basic Existence Theorem . . . . . . . . . . . . . . 203

E.2 Homogeneous Linear Differential Equations . . . . . . . . 203

E.2.1 Linear Combination . . . . . . . . . . . . . . . 204

E.2.2 Linear Dependent and Linear Independent

Functions . . . . . . . . . . . . . 204

E.2.3 Theorems on Linear Second-Order Homogeneous

Differential Equations . . . . . . . . . . . . 204

E.3 Inhomogeneous Linear Differential Equations . . . . . . . 205

E.3.1 Principle of Superposition . . . . . . . . . 206

E.3.2 Solutions of Linear Inhomogeneous Differential Equations . . . . . . . . . . . 207 
E.4 Linear Second-Order Homogeneous Differential Equations with Constant Coefficients . . . . . . . . . . . . . 207

E.5 Linear Second-Order Inhomogeneous Differential Equations with Constant Coefficients . . . . . . . . . . 208

E.6 Secular Terms . . . . . . . . . . . . . . . . . . . . 210 References .................... 211

Appendix F Lindstedt-Poincaré Perturbation Method 213

References . . . . . . . . . . . . . . . 216

Appendix G A Standard Averaging Method 217

References ................... 220

Appendix H Discrete Models of Two TNL Oscillators 221

H.1 NSFD Rules . . . . . . . . . . . . . . . . . . . 221

H.2 Discrete Energy Function . . . . . . . . . . . . . 222

H.3 Cube-Root Equation . . . . . . . . . . . . . . . . . . 223

H.4 Cube-Root/van der Pol Equation . . . . . . . . . . . 225

References ..................... 226

$\begin{array}{ll}\text { Bibliography } & 227\end{array}$

$\begin{array}{ll}\text { Index } & 237\end{array}$ 
This page intentionally left blank 


\section{List of Figures}

2.2.1 Basic properties of the phase-plane for the linear harmonic oscillator. The dashed-line (- - ) is the "zero" null-cline, the solid line $(-)$ is the "infinite" null-cline. The $( \pm)$ indicates the sign of $d y / d x$ for the designated region. . . . . . . . . . . .

2.2.2 Geometric proof that phase-plane trajectories are closed curves using the symmetry transformations. . . . . . . . . . 30

2.3.1 $\ddot{x}+f(x)=-\epsilon g(x) \dot{x}$ : (a) Periodic solutions for $\epsilon=0$. (b) Damped oscillatory solutions for $\epsilon>0 \ldots \ldots \ldots$

2.3.2 Phase-plane for Eq. (2.3.15). The dashed lines are the $y_{0}(x)$ null-clines. The solid line is the $y_{\infty}(x)$ null-cline. . . . . . 38

2.3.3 Typical trajectories in the phase-plane for Eq. (2.3.17). . . . . 38

3.2.1 (a) Phase-plane for $\ddot{x}+x^{-1 / 3}=0$. Vertical dashes denote the infinite null-cline, $y_{\infty}(x)$. (b) Trajectory passing through $x(0)=$ $A$ and $y(0)=0 \ldots \ldots \ldots \ldots \ldots \ldots$

5.2.1 Plot of $\Omega^{2}(A)$ versus $A$ for the periodic solutions of Eq. (5.2.118) . . . . . . . . . . . . . . 113

6.2.1 Schematic representation of the solution for $\ddot{x}+x=-2 \epsilon(\dot{x})^{1 / 3}$.

7.4.1 Plot of $\Omega(t, A, \epsilon)$ versus $t$, for the linearly damped, pure cubic Duffing equation. $\Omega_{0}(A)=\left(\frac{3}{4}\right)^{1 / 2} A \ldots \ldots \ldots \ldots 4$

7.4.2 Plot of the numerical solution of $\ddot{x}+x^{3}=-2 \epsilon \dot{x}$ for $\epsilon=0.01$, $x(0)=1$ and $\dot{x}(0)=0 \ldots \ldots \ldots \ldots \ldots \ldots$

7.5.1 Plot of the numerical solution of $\ddot{x}+x^{1 / 3}=-2 \epsilon \dot{x}$ for $\epsilon=0.01$, $x(0)=1$ and $\dot{x}(0)=0 \ldots \ldots \ldots \ldots \ldots$ 
7.5.2 This graph is the same as that in Figure 7.5.1, except that the interval in time is twice as long. . . . . . . . . . . . . 169

7.6.1 Numerical solution of Eq. (7.6.1) for $x(0)=4, y(0)=0$, and $\epsilon=0.1 \ldots \ldots \ldots \ldots \ldots \ldots \ldots \ldots \ldots \ldots$

7.6.2 Numerical solution of Eq. (7.6.2) for $x(0)=0.1, y(0)=0$, and $\epsilon=0.1 \ldots \ldots \ldots \ldots \ldots \ldots \ldots \ldots \ldots$ 


\section{List of Tables}

3.2 .1 Values for $A^{1 / 3} \Omega(A) \ldots \ldots \ldots \ldots \ldots \ldots$

7.4.1 Comparison of the amplitude and effective angular frequencies for the linearly damped, pure cubic, Duffing oscillator. . . . . . 167 